\title{
On the Multivariate Extremal Index
}

\section{S. Nandagopalan \\ Colorado State University, Fort Collins, CO $80 \$ 23$}

\author{
The excedance print process apprach of \\ Hsing el al. is extended to multivariate \\ stationury sequences and some weak con- \\ vergence results are oblained. It is welt \\ known that under general mixing assump- \\ tions, high level exceedances typitully \\ have a limiting Compound Poisson structure \\ whene mulliple events are caused by the \\ clustering of exccedances. In this paper we \\ explore (a) the precise effect of such \\ clustering on the limil, and (h) the relation- \\ ship between point process convergence \\ and the limiting behavior of maxina. Fol-
}

lowing this. the notion of nullivariale extremal index is introduced which is shown to have propertics analogous to its uni= variale counterparl. Two examples of bivariate moving average sequences are presented for which the extremal index is calculated in some special cases.

Kry words: dependence function; ex = ceedance; exlremal index: multivariale: point process; stationary.

Accepted: March 22, 1994

\section{Introduction}

Extreme value theory for multivariate iid sequences has been studied for quitc some time now but attention to the dependent case has boen relatively recent. For univariate sequences it is known that local dependence causes extreme values to occur in clusters, which in turn results in a stochastically smalker distribution for the maximum than if the observations were independent. We begin with a brief review of these results, which we shall later extcrid to the multivariate casc.

Let $\left\{\xi_{n}\right\}$ be a univariate stationary sequence. Write $M_{n}=\max \left\{\xi_{1}, \ldots, \xi_{n}\right\}$ and for $\pi=0$, let $\left\{u_{n}(\tau)\right\}$ denotc al sequence satisfying $\lim _{n \rightarrow \infty} n P\left\{\xi_{1}>u_{n}(\tau)\right\}=\tau$. Under quite general mixing assumptions there exist constants $0 \leq \theta^{\prime} \leq \theta^{\prime \prime} \leq 1$ such that

$$
\begin{aligned}
& \limsup _{n \rightarrow \infty} P\left\{M_{n} \leq u_{n}(\tau)\right\}=c^{\theta t} \text { and } \\
& \liminf _{n \rightarrow x} P\left\{M_{n} \leq u_{n}(\tau)\right\}=\mathrm{e}^{-f^{n} \tau}
\end{aligned}
$$

for all $\tau$. (See Ref. [1], although the idea actually dates back to Refs. [2-4].) Thus if $P\left\{M_{n} \leq n_{n}\left(\right.\right.$ To $\left.\left._{0}\right)\right\}$ converges for some $\tau_{0}$. then $\theta^{\prime}=\theta^{\prime \prime}(=\theta$, say $)$ and hence $\lim _{n \rightarrow \infty} P\left\{M_{n} \leq u_{n}(\tau)\right\}=c^{-\theta r}$ for all $\tau>0$. The common value $\theta$ is then called the extremal index of $\left\{\xi_{t}\right\}$. We shall assume $\theta$ to be positive whenever it exists. since the case $\theta=0$ corresponds to a degeneratc limiting distribution for $M_{n}$. Note that $\theta=1$ for iid sequences. Let $\left\{\hat{\xi}_{n}\right\}$ be an iid sequence with $\hat{\xi}_{l}={ }^{d} \xi_{1}$, called the associated iid sequence, and writc $\hat{M}_{n}=\max \left\{\hat{\xi}_{1} \ldots, \hat{\xi}_{n}\right\}$. If $\left\{\xi_{n}\right\}$ has extremal index $\theta$ and $\lim _{n \rightarrow \infty} P\left\{M_{n} \leq v_{n}(t)\right\}=H(t)$ for a suitablc family of normalizing constants $\left\{v_{n}(t)\right\}$, then it follows (upon identifying $\mathrm{e}^{-\theta \tau}$ with $H(t)$ ) that $\lim _{n \rightarrow x}$ $P\left\{\hat{M}_{n} \leq v_{n}(t)\right\}=\hat{H}(t)$ where

$$
H(t)=\hat{H}(\mathrm{r})^{*} .
$$

The extremal index is thus a measure of the effect of 
dependence on the liniting distribution ot' $M_{4}$. 'The stochastically smaller limiting distribution of $M_{n}$ is in fact a direct result of the clustering of extremes, as explained below. See Ref. $\lceil 5\rceil$ for detalls.

For fixed $\rightarrow 0$ let the excedance pom process $N_{n}=N_{n}^{(r)}$ be delined by

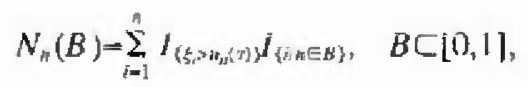

where $I_{A}$ denotes the indicator furction of the event $A$. Then for a broad class of weakly dependent sequences, the limit in distribution of $N_{m}$, if it cxists, is a Compound Poisson process with intensily $\theta_{r}$ and ruultiplicity distrbution $\pi$ on $\{1,2, \ldots\}$. "Ihe Poisson events may in fact be rcyarded as the positions of "cxccedance clusters" whilc the multiplicitics correspond to cluster sizes. More explicitly, one maty divide the $n$ observations into $k_{n}$ blocks of roughly cyual sizc and regard cxccedances within each block as forming a single "cluster" , so that the cluster sizes are given by $N_{n}\left(J_{i}\right), i=1, \ldots, k_{n}$, where $J_{r}\left(-J_{n, j}\right)=\left(\frac{i-1}{k_{n}}, \frac{i}{k_{n}}\right)$. Fin a suitable choice of $k_{n}$ depending on the mixing rate of $\left\{\xi_{t}\right\}$, one then has

$$
\lim _{n \rightarrow \infty} p\left\{N_{n}\left(J_{1}\right)=j \mid N_{n}\left(J_{1}\right)>0\right\}-n(j), \quad j \geq 1
$$

and

$$
\begin{gathered}
\lim _{n=n=\infty} P^{k_{n}}\left\{N_{n}\left(J_{1}\right)-0\right\}=\lim _{n \rightarrow \infty} P\left\{N_{n}[0,1]-0\right\} \\
=\lim _{n \rightarrow \infty} P\left\{M_{n}=u_{n}(\tau)\right\}=\mathrm{c}^{-0, \pi}
\end{gathered}
$$

so that in particular, $\lim _{\mu \rightarrow \mathrm{n}} k_{\mu} \mu\left\{N_{n}\left(J_{1}\right)>0\right\}=\theta \pi$. Hence

$$
\begin{gathered}
\lim _{n+\infty} E N_{n}|0,1|=\lim _{n \rightarrow \infty} k_{n} F N_{n}\left(J_{1}\right) \\
=\lim _{n \rightarrow \infty} k_{n} E\left(N_{n}\left(J_{1}\right) \mid N_{n}\left(J_{1}\right)>0, \Gamma^{2}\left\{N_{n}\left(J_{1}\right)>0\right\}\right. \\
=\theta \tau \lim _{n \rightarrow \infty} E\left(N_{n}\left(J_{1}\right) \mid N_{n}\left(J_{1}\right)>(1),\right.
\end{gathered}
$$

while on the other laand, $\operatorname{lin}_{n \rightarrow-} E N_{n}\left[0, ! \mid=\lim _{n \rightarrow 1}\right.$. $n P\left\{\xi_{1}>u_{n}(\tau)\right\}=\tau$. The cluster size distribution and the extremal index are therefore related by

$$
\operatorname{limin}_{n \rightarrow x} E\left(N_{n}\left(J_{1}\right) \mid N_{n}\left(J_{1}\right)>0\right)=1 / \theta
$$

Now let $\left\{\xi_{n}=\left(\xi_{n}, \ldots, \xi_{n d}\right), n \in \mathbb{Z}\right\}$ be a multivariate sta lionary sequence wlicrc $d \geq 1$ is a fixed integcr, and write $M_{n}=\left(M_{n 1}, \ldots, M_{n+1}\right)$ where $M_{n j}=\max \left\{\xi_{1}, \ldots, \xi_{n,}\right\}$, $j=1_{n+\ldots}, d$. The study of multivariate extremes began in the early 1950 s, focusing mainly on the limiting behavjor of $M_{n}$ under a lincar normalization, when the observations are iid. The resulting class of limiting distribulions was charaterized in Ref. [6] and Jonuains of attraction criteria were given in Rel" [7]. See also Ref.
[8]. Chapter 5 , for an account of the literature surrounding this theory. For stationary sequences satisfying a general mixing assumption, it is known (see Refs. 19 , 107 , and Theorem 1.1 below) that the class of limiting distributions of $M_{n}$ is the same as for iid sequences. In this paper we explorc the precise effect of dependence on the limiting distribution by cxtending the univariate theory dexcribed abowe to the multivariate casc. Essentially, this involves studying the intcr-relationship between the two dependence structures present, onc duc to dependence over time and the other due to the dependence betwien the varius components of the multivariatc observations. The idcas beconc most transparent when presented in terms of so-called dcpendence functions [8]. Here we adopt the slightly moditied definition found in Ref. [9]. A distribution function $D$ on $[0,1]^{d}$ is called a dependence function if $D_{j}\left(D_{j}(u)\right)=D_{j}(u)$, $u \in[0,1], j=1, \ldots, d$, wherc the subseript $j$ significs the $j$ th marginal. The dependence function of a distribution $F$ on IR ${ }^{d}$ is defined by $D_{r}(u)=P\left\{F_{1}\left(X_{1}\right) \leq\right.$

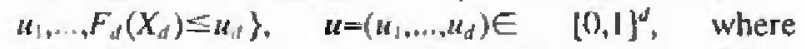
$\left(X_{1, \ldots}, X_{d}\right)$ is a randort vector with distribution $F$. Morc generally, any dependence function satisfying $F(x)=D\left(F_{1}\left(x_{1}\right)_{4} \ldots, F_{d}^{2}\left(x_{d}\right)\right)$ coult be defined to be a deperkience function of $F$, although the present choice is at Matural onc.

Write $T=(0,1)^{\text {th }}\{1\}$ where $I=(1, \ldots, 1) \in \mathbb{I R}^{d}$, and for $\mathbf{t}=\left(t_{1}, \ldots t_{d^{\prime}}\right) \in T$, let $v_{n}(\mathrm{t})=\left(v_{n d}\left(t_{1}\right), \ldots, v_{n d}\left(t_{d j}\right)\right)$ where $v_{n j}\left(t_{j}\right)$ satisfics $\lim _{n \rightarrow \infty} n P\left\{\xi_{1}>y_{n j}\left(I_{j}\right)\right\}=-\log l_{j}$. Let $H_{n}$ denote the distribution function of $M_{\mathrm{t}}$ (i.e., $\left.H_{n}(x)=P\left\{M_{n} \leq x\right\}\right)$ ). with marginals $H_{n j}, j=1, \ldots, d$. Then (sce Refs. $[8,11]$ ).

$$
H_{n}\left(u_{n}(\mathrm{t})\right) \rightarrow \cdots(\mathrm{t})
$$

if and only if

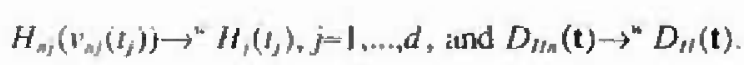

The limiting behavor of $\boldsymbol{M}_{\text {a }}$ can thereforc be separated into two parts, one pertaining to the convergence of the marginals (a univariate problem) and the other to the convergence of the dependence functions. Here we focus attention cxclusively on the latter. It should bx: noted that the choice of normalising constants does not affect the dependence function of the limit distribution $H$, but only alters the marginals (sce Ref. 191, Lemma 3.2). Since our main interest is in the dependence function. the present choice of normalising constants is appropriate in vicw of the fact that it results in Uniform[0,1] malginals for the limit distribution when $\left\{\xi_{n}\right\}$ is iid, so that in particular $D_{H}=/ I$. According to Theorcm 3.3 of Ref. 19$]$, the class of all possible limits $H$ in Eq. (3) (for iid $\left\{\xi_{n}\right\}$ is precisely the class of extreme dependence functions, that is those that satisfy 


$$
D^{n}(\mathrm{t})=D\left(t_{1}^{n} \ldots, \ldots, t_{i}^{n}\right)
$$

for each $n>1$ and $t=\left(t_{1}, \ldots, t_{d}\right) \in[0,1]^{d}$. Theorem 1.1 bclow shows that the same is truc also if $\left\{\xi_{n}\right\}$ is a stationary scquence satisfying the following mixing condition.

For $\mathbf{t} \in T$, let

$$
\operatorname{SB}_{k}^{\prime}\left(v_{n}(\mathbf{t})\right)=\sigma\left\{\left(\xi_{i p}>v_{n j}(t)\right): k \leq i \leq l, j=1, \ldots, d\right\}
$$

and for $1 \leq l \leq n-1$, define

$$
\begin{gathered}
\alpha_{n},-\sup \left\{|P(A \cap B)-P(A) P(B)|: A \in \mathscr{B}_{1}^{k}\left(v_{n}(\mathbf{t})\right),\right. \\
\left.B \in \mathscr{B}_{k+1}^{n}\left(v_{n}(\mathbf{t})\right), \mid \leq k<k+l \leq n\right\} .
\end{gathered}
$$

The mixing condition $\Delta\left(v_{n}(t)\right)$ is then said to hold if $\alpha_{n d_{n}} \rightarrow 0$ for some sequence $\left\{l_{n}\right\}$ sat isfying $l_{n} / n \rightarrow 0$. This is the multivariate version of the mixing condition uscd in Ref. [5] and is slightly stronger than the $D\left(k_{n}\right)$ condition in Ref. [9]. Henceforth $\left\{\xi_{n}\right\}$ will be assumed to satisfy $\Delta\left(v_{n}(\mathbf{t})\right)$, for some or all $\mathbf{t}$, as required.

THEOREM 1.1. Let $\left\{\xi_{n}\right\}$ satisfy $\Delta\left(v_{n}(\mathbf{t})\right)$ for all $\mathbf{t} \in T$ and suppose that $P\left\{M_{n} \leq v_{n}(t)\right\} \rightarrow " H(t)$, non-degenerate. Then $D_{n}$ is an extreme dependence function and hence, in particular, $H(\mathbf{t})=H^{c}(\mathbf{t})$ for each $\mathbf{t} \in[0,1]^{d}$ and $c>0$ (where $\mathfrak{t}^{c}=\left(t_{\Gamma}^{c}, \ldots, t_{d j}^{c}\right)$ ).

Proor: The first part is an immediatc consequence of Theorem 4.2 of Ref. [9] while the second part follows from the definition of extreme dependence functions upon noting that (by the univariate theory described above), the marginals of $H$ are of the form $H_{j}\left(t_{j}\right)=t_{j}{ }_{j}$ where $\theta_{j}$ is the extremal index of $\left\{\xi_{n j}\right\}$, the $j$ th-component sequence of $\left\{\xi_{n}\right\}$.

In the next section we apply the cxccedance point process approach to multivariate extremes and obtain some weak convergence results. The multivariate extremal index is then defined (in Sec. 3). based on the multivariate analogue of Eq. (1). It is seen to be a function of only $d-1$ variables and its properties naturally extend those of the univariate extremal index. Finally in Sec. 4 we consider two examples of bivariate moving average sequences for which the computation of the extremal index is demonstrated.

\section{Exceedance Point Processes}

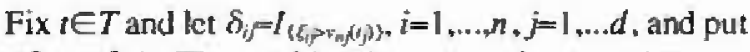
$\delta_{i}=\left(\delta_{i 1}, \ldots, \delta_{i d}\right)$. The multivariate exceedance point process $N_{n}=N_{a}^{(t)}$ is then defined by

$$
N_{n}(B)=\sum_{i=1}^{n} I_{\text {iñ } n \in \cap,}, \delta_{1}, \quad B \in[0,1]
$$

Assume that $\left\{\xi_{n}\right\}$ satisfies $\Delta\left(v_{n}(\mathbf{t})\right)$. If also $N_{n} \rightarrow^{d} N_{t}$, then it may be shown (as in the univariate cuse) that the limit $N_{0}$ is a point process on $[0,1]$ which is of Compound Poisson type. More precisely, the Laplace Transform of $N_{u}$ is given by

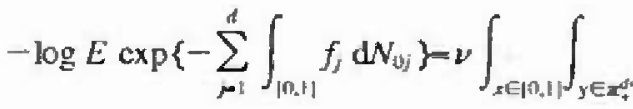

$$
\begin{aligned}
& \left(1-\exp \left\{-\sum_{j=1}^{d} y_{j} f_{j}(x)\right\}\right) \mathrm{d} \pi(y) d x \text {. }
\end{aligned}
$$

Here $N_{0 j}$ denotes the jth-component of $N_{0}, \nu$ is a positive constant, $\pi$ is a probability distribution on $\mathbb{Z}_{+}^{d A}=\{0,1,2, \ldots\} \backslash\{0\}$ and $f_{f}{ }^{\prime} s$ are non-ncgative functions on $[0,1]$. ing

Let $\left\{k_{n}\right\}$ be any sequence of positive integers satisfy-

$$
k_{n} \rightarrow x, k_{n} l_{n} / n \rightarrow 0 \text {, and } k_{n} \alpha_{n, l_{n}} \rightarrow 0 \text {, as } n \rightarrow \infty \text {. }
$$

Set $r_{\mathrm{n}}=\left[n / k_{n}\right]$ (the largest integer not excecding $n / k_{n}$ ) and put $J_{n 1}=\left[0, r_{n} / n\right]$. Define the probability distribution $\pi_{*}$ on $Z_{*}^{d s}$ by

$$
\pi_{n}(y)=P\left\{N_{n}\left(J_{a 1}\right)=\mathrm{y} \mid N_{n}\left(J_{n 1}\right) \neq 0\right\}, \mathrm{y} \in \mathbb{Z}_{+}^{d r} .
$$

The following theorem which gives a uscful characterization of the convergence of $N_{n}$ is an immediatc consequence of the results in Sec. 5 of Ref. [12].

THEOREM 2.1, $N_{n} \rightarrow^{d} N_{0}$ if and only if $\pi_{n} \rightarrow^{\omega *} \pi$ and $P\left\{M_{n} \leq v_{n}(\mathrm{t})\right\} \rightarrow \mathrm{e}^{-v}$. and in that case the Laplace Transform of $N_{0}$ is given by Eq. (6).

Next we consider the iid case in some detail and oblain an interesting connection with Theorem 5.3.1 of Ref. [8].

Prolosition 2.2. Let $\left\{\xi_{n}\right\}$ be iid and for fixed $\mathrm{t} \in T$ let $N_{n}$ be defined by Eq. (5). If $N_{n} \rightarrow{ }^{4} N_{0}$ then the multiplicity distribution $\pi$ in Eq. (6) is supported on the set $S=\{0,1\} \backslash\{0\}$.

Proof: Observe that $\Delta\left(w_{n}(t)\right)$ is trivially satisfied since $\alpha_{n, 1}=0$ so that we may take $l_{n} \equiv 1$ and $k_{n}=n$. Then $\pi_{n}(\mathbf{y})=P\left\{\delta_{1}=\mathbf{y} \mid \xi_{1} \pm v_{n}(\mathbf{t})\right\}, \mathbf{y} \in \mathbb{Z}_{*}^{d^{\prime}}$, which is clearly supported on $S$. The result is now immediate since $S$ is a closed set and $\pi_{n} \rightarrow^{n} \pi$ by Theorem 2.1.

Making the dependence on $\mathbf{t}$ explicit, we now write $N_{n}=N_{n}^{(t)}, N_{1}=N_{0}^{(t)}, \nu=\nu^{(t)}$ and $\pi=\pi^{(t)}$. In addition we shall 
require the following notation from Ref. [8]. For $1 \leq k \leq d$, let $\mathbf{j}(k)=\left(j_{1}, \ldots, j_{k}\right)$ denote a vector with integervalued components $1 \leq j_{1}<j_{2}<\cdots<j_{k} \leq d$, and for

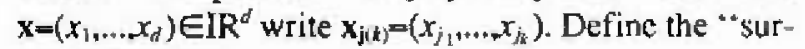
vival function"

$$
G(\mathbf{x})=P\left\{\xi_{11}>x_{1}, \ldots, \xi_{1,1}>x_{d}\right\}
$$

and write $G_{j(h)}(\mathbf{x})=P\left\{\xi_{1, j}>x_{j_{1}}, \ldots \xi_{1, j_{k}}>x_{j_{k}}\right\}$. For each $\mathrm{j}(k)$, let $\mathrm{y}_{\mathrm{j}(k)}$ denote the element in $\left.S=\{0.1\} \backslash 0\right\}$ whose $j$ th component equals 1 if and only if $j=j_{i}$ for some $i=1, \ldots, k$. (This defines a natural 1-1 correspondence between $S$ and the $\mathrm{j}(k)$ 's.)

THFoжem 2.3. Let $\left\{\xi_{n}\right\}$ be iid. Then $N_{0}^{(1)} \rightarrow^{d} N_{n}^{(n)}$ for some flued $\mathrm{t} \in T$ if and only if

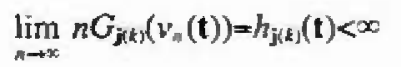

for each $\mathrm{j}(k), 1 \leq k \leq d$. In that case $N_{0}^{(1)}$ has Laplace Transform given by Eq. (6) with

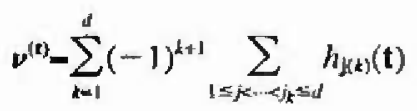

and with $\pi^{(0)}$ desermined by the relations

$$
h_{\mathbf{j}(t)}(\mathbf{t})=\nu^{(1)} \sum_{\mathbf{y} \geq y_{j(t)}} \pi^{(t)}(\mathbf{y})
$$

PROOF: Write $S_{k}(t)=\Sigma_{\mid \Sigma_{j}<-\sum_{h} \leq d} G_{j(k)}(t)$, so that $P\left\{\xi_{1} \equiv\right.$ $\left.v_{n}(t)\right\}-\sum_{k=1}^{d}(-1)^{k+1} S_{k}\left(v_{n}(t)\right)$. If Eq. (8) trolds for each $k$, then

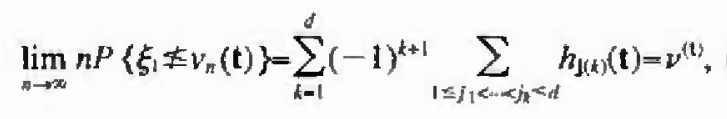

and hence $\operatorname{Tim}_{n \rightarrow I} P\left\{M_{n} \leq v_{n}(t)\right\}=\mathrm{e}^{-\nu^{(n)}}$.

Next observe that for each $\mathrm{j}(k) .1 \leq k \leq d$.

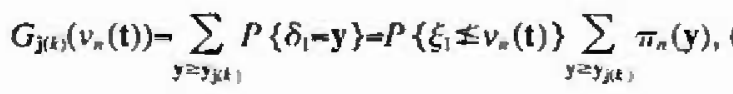

where $\pi_{n}(\mathbf{y})=P\left\{\delta_{1}-\mathbf{y} \mid \xi_{1} \notin v_{n}(\mathbf{t})\right\}$. Moreover, this relationship is invertible in the sense that each of the probabilities $\pi_{n}(\mathbf{y}), y \in S$, can be expressed as a linear combination of the $G_{(k)}\left(v_{n}(t)\right)$ 's. Thercfore by Eq. (8). $\lim _{n \rightarrow \infty} \pi_{n}(y)-\pi^{(t)}(y)$ (say) exists and satisfies Eq. (10). Hence by Theorem $2.1 N_{n}^{(t)} \rightarrow^{d} N_{0}^{(t)}$ wherc $N_{n}^{(t)}$ has the specified parameters. Converscly if $N_{m}^{(t)} \rightarrow{ }^{d} N_{0}^{(t)}$ then $\pi_{n}$ and $P\left\{M_{n} \leq v_{n}(t)\right\}$ converge (by Theorem 2.1 again), and hence Eq. (8) follows by virtue of Eqs. (11) and (12).
COROLlary 2.4. Let $\left\{\xi_{n}\right\}$ be iid. Then $N_{n}^{(1)} \rightarrow^{(t} N_{0}^{(1)}$ for each $\mathbf{t} \in T$ if and only if $\mathrm{P}\left\{M_{n} \leq v_{n}(\mathbf{t})\right\} \rightarrow^{n-H}(\mathbf{t})$. More over $H$ and $\left\{\nu^{(t)}, \pi^{(t)}\right\}_{\in \in T}$ determine each other.

PROOF: (Sketch) The first part follows from Theorem 2.3 above and Theorem 5.3.1 of Ref. [8] which states that $P\left\{M_{n} \leq \nu_{n}(\mathrm{t})\right\} \rightarrow^{*} H(\mathbf{t})$ if and only if Eq. (8) holds for each $\mathrm{t} \in T$. Note that $H(\mathrm{t})=\mathrm{e}^{-2(t)}$ so that $H$ and the $\nu^{(1)}$ 's can be obtained from each other. Also the $\pi^{(t)} \mathrm{s}$ can be obtaincd from the $\nu^{(1)}$ 's by first inverting Eq. (9) to get the $h_{j(k)}(t)$ 's and then inverting Eq. (10). (The inversion of Eq. (9) is carried out inductively using the fact that the weak convergence of $H_{n}\left(v_{n}(t)\right)$ implies that of all lower dimensional marginals.) $\square$

Analogous results for the dependent case take on a slightly different form. Let $\left\{\xi_{n}\right\}$ be a stationary sequence satisfying $\Delta\left(v_{n}(\mathbf{t})\right)$ for each $\mathbf{t} \in T$. As before let $r_{R}=\left[n / k_{n}\right]$ where $\left\{k_{n}\right\}$ is any sequence satisfying Eq. (7). and define

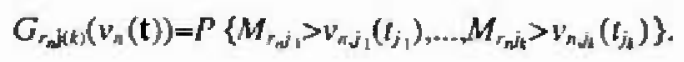

THEOREM 2.5. Let $\left\{\xi_{n}\right\}$ be a stationary sequence satisfying $\Delta\left(v_{n}(t)\right)$ for each $\mathbf{t} \in T$. Then $P\left\{M_{n} \leq v_{n}(\mathbf{t})\right\} \rightarrow^{w} H(\mathbf{t})$ if and only if

$$
\lim _{n \rightarrow \infty} k_{n} G_{r_{n} j(k)}\left(v_{n}(t)\right)=h_{j_{j(k)}}(t)<x
$$

for each $\mathrm{j}(k), 1 \leq k \leq d$ and $\mathrm{t} \in T$, and in that case

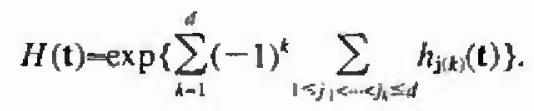

Proof: Observe that the mixing condition $\Delta\left(v_{n}(t)\right)$ implics that $\left\{\xi_{n j(k, k}\right\}$ satisfies $\Delta\left(v_{n j(k)}(t)\right)$ for each $\mathbf{j}(k)$ (with obvious notation). Hence it may be shown as in the univariate case (see Lcmma 2.1 of Ref. (11) that

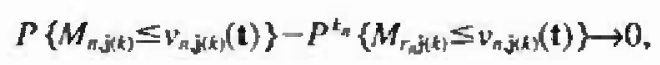

for each $\mathbf{j}(k)$. The rcsult may therefore be proved in exactly the same way as Theorem 5.3.1 of Ref. [8].

REMARK: Under the hypothesis of Theorem 2.5, if $N_{n}^{(t)} \rightarrow^{d} N_{i t}^{(t)}, \mathbf{t} \in T$, with paramcters $\nu^{(1)}$ and $\pi^{(t)}$ then $P\left\{M_{n} \leq v_{n}(\mathbf{t})\right\} \rightarrow^{*} H(\mathbf{t})-\mathrm{c}^{-p^{(t)}}$, as in the iid casc. However it is not possible in general to recover the $\pi^{(1)}$ 's from $H$ since the clustering of exceedances may cause the support of $\pi^{(t)}$ to extcnd beyond $S$. References $[9,10]$ give sufficient conditions (analogous to the $D^{\prime}\left(u_{n}\right)$ condition of Ref. [13]) under which clustering does not occur, so that Corollary 2.4 can be extended to stationary sequences satisfying this condition. 
A distribution function $F$ on $\mathrm{IR}^{d}$ is said to be imdepertdent if $F(\mathbf{x})=\left.1\right|_{j=1} ^{d} F_{j}\left(x_{j}\right), \mathbf{x} \in \mathrm{IR}^{d}$. If $\left\{\xi_{n}\right\}$ is iid and $P\left\{M_{n} \leq v_{n}(\mathrm{t})\right\} \rightarrow^{*+} H(\mathrm{t})$. then it follows from (oullaty 5.3.1 of Ref. [8] that $H$ is independent if and only if the marginals of $H$ are pairwise independent. The analigins result for the dependent case is stated beliow. The prisst (which is omitted) is cssentially the sane as for the ini case, but uses Theorem 2.5 instcad of Theirent 5.3 .1 it Ref. [8].

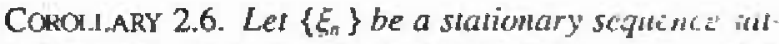
isfying $\Delta\left(v_{n}(t)\right)$ for each $\mathrm{t} \in T$ and suppose the $P\left\{M_{n} \leq v_{n}(\mathbf{t})\right\} \rightarrow{ }^{*} H(\mathbf{t})$. Then $H$ is independent if and only if $k_{A} P\left\{M_{r,}>v_{n, j}\left(t_{i}\right), M_{r,}>v_{A, j}\left(t_{i}\right)\right\} \rightarrow 0$ for each $I \leq j<l \leq d, \mathrm{t} \in T$, i.e., if and only if $k_{n} G_{r, j(t)}\left(v_{n}(\mathrm{t})\right) \rightarrow 0 \mathrm{for}$ each $\mathrm{j}(2)$ and each $\in \mathbb{E}$.

It is shown in Ref. [14] that $H$ is independent if $H(\mathbf{t})=\prod_{j=1}^{d} H_{j}\left(t_{j}\right)$ for some $t \in(0,1)^{d}$. Althergh the result in [14] only stated for iid scquences under a linear uor . malization, the prof essentially rests on the defining property of extreme dependence functions, nanely $\mathrm{Eq}$. (1). Consequently the result cxtends to the preseul nurgre general situation allowing dependence and non-linedr normalizations. Corollary 2.6 can therefore be improved as follows.

COROLlary 2.7. Let $\left\{\xi_{n}\right\}$ be as in Corollary 2.0 and suppose that $P\left\{M_{n} \leq v_{n}(t)\right\} \rightarrow^{w \prime} H(\mathbf{t})$. Then the following are equivalent:

(i) $H$ is independent,

(ii) $H(\mathrm{t})=-\mathrm{T}_{j=1}^{d} H_{j}\left(t_{j}\right)$ for some $\mathrm{t} \in(0,1)^{d}$.

(iii) $k_{n} G_{r, j(k)}\left(v_{a}(\mathbf{t})\right) \rightarrow 0$ for each $\mathbf{j}(2)$. for sone $\mathbf{t} \in(0,1)^{d}$.

It should be noted that Refs. $[9,10]$ give some interesting sufficicnt conditions for $H$ to be indeperident when $\left\{\xi_{n}\right\}$ is a stationary sequence. A natural question to ask in the present context is whether $H$ is independent whenever $\hat{H}$ is. Proposition 3.4 gives a necessary ard sufficient condition for this in ternus of the extremal index, but the answer in general is negative and a coun ter-example can be found in [10]. It scems norc plansi. ble that the converse may be true, i.c., that $\hat{H}$ is independent whenever $H$ is. In fact however, this too is not the case, as shown by an intercsting countci-example in [15].

We conclude this section by stating a rcsult which extends Theorem 5.1 of [5] and is proved similarly.

THEORFM 2.8. Let $\left\{\xi_{n}\right\}$ be a srationary seguente satis. fying $\Delta\left(v_{n}(\mathbf{t})\right)$ for each $\mathbf{t} \in T$ and suppose that $N_{n}^{(\mathbf{t})}-i^{t} N_{01}^{\text {rit }}$ for some $\mathrm{t} \in T$. Then $N_{n}^{(1)} \rightarrow{ }^{d} N_{0}^{(r)}$ for each $c>0$ and fur

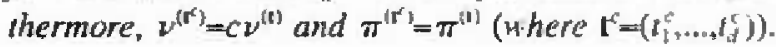

\section{The Multivariate Extremal Index}

1 at $\left\{\xi_{\text {s }}\right\}$ be a stathonaty sequence and $\left\{\hat{\xi}_{n}\right\}$ the associared idd seciuence. Suppose that $P\left\{M_{n} \leq v_{n}(\mathrm{t})\right\} \rightarrow{ }^{w} H(\mathbf{t})$ and $F^{\prime}\left\langle\hat{M}_{n} \leq y_{a}(t)\right\} \rightarrow^{n \cdot} \hat{H}(\mathbf{t})$. 'The multivariate extremal inder of $\left\{\xi_{i}\right\}$ is then defined by the relation $H(t)-H^{\text {th }}$ (1) (sce Eu. (1)), or more explicitly

$$
\theta(\mathrm{t})=\log H(\mathrm{t}) / \log \hat{H}(\mathrm{t}), \quad \mathrm{t} \in T \text {. }
$$

(A) Hezh that $\theta(\mathbf{t})$ is well defined since $\hat{H}$ has Unifor $m[0,1]$ intiginals and hence, $0<\hat{H}(t)<1$ on $T$. The following results tescribe some basic propertics of the multivariate extremal index.

l'KOPUSI IKiv 3.1. Assume that $\left\{\xi_{n}\right\}$ satisfies $\Delta\left(v_{n}(t)\right)$ for each $\mathbf{t} \in T$ and has extremal index $\theta(\mathbf{t})$. Then

(i) $\theta(\mathbf{t})=\theta(\mathbf{t})$ for each $\mathbf{t} \in T$ and $c>0$, and

(ii) fon each $j=1, \ldots, d,\left\{\xi_{n j}\right\}$ has extremal index $\theta_{j}=\theta(t)$ where $t E T$ has all coordinates equal to l except the jith.

(Noice that by (i). $\theta(t)$ is constant along the contours $L_{i}<\left\{t^{i}:<>0\right\}, \mid \in T$, and hence $\theta_{j}$ in (ii) is well-defined.)

Ifout: Kecall that (by Theorem 1.1) $H\left(t^{\circ}\right)=H^{c}(\mathbf{t})$ and $\dot{H}\left(\mathrm{t}^{c}\right)=\dot{H}^{\prime}(\mathrm{t})$ so that $(j)$ follows from the definition of the extremal irdex. Next, for $t \in T$ with all coordinates but the $j$ th equal to $1, P\left\{M_{n} \leq v_{n}(t)\right\}-P\left\{M_{n j} \leq v_{m j}\left(t_{j}\right)\right\}$ and hence

$$
\lim _{n \rightarrow \pi} P\left\{M_{n j} \leq v_{n j}\left(l_{j}\right)\right\}-\lim _{n \rightarrow \infty} P\left\{M_{n} \leq b_{n}(\mathbf{t})\right\}=H(\mathbf{t})=H_{j}\left(t_{j}\right) .
$$

Thejcfure by Theoten 2.2 of Ref. [11. $\left\{\xi_{n j}\right\}$ has extremal index $\theta_{j}$ (say) so that $H_{j}\left(t_{j}\right)=t t_{j}^{\theta_{j}}$. Now $\|(t)=H^{k t)}(t)$ by definition of the extremal index, and for the Juresent choice of $t$ this is the same as $H_{j}\left(t_{j}\right)=t_{j}^{6,1)}$, whence it fiollows that $\theta(\mathbf{t})=\theta_{j}$ for all such $\mathbf{t}$.

For $t \in T$, let $\mathcal{N}_{n}^{(t)}$ clenote the onc-dimensional point process obtaincul fron $N_{t}^{(t)}$ via the map $\mathrm{y} \rightarrow l_{\mathrm{y} \neq 0\}}$ from

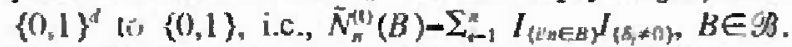
Thus $N_{n}^{(t)}$ has unit rnass at $i / n$ if and only if $\xi_{n} \leftleftarrows v_{n}(t)$. Assume that $\left\{\xi_{n}\right\}$ satisfics $\Delta\left(v_{n}(t)\right)$ and with $J_{n 1}$ as in Sec. 2. let

$$
\left.\bar{\pi}_{n}^{(1)}(y)=P\left\langle\hat{N}_{n}^{(1)}\left(J_{n 1}\right)=y\right| \bar{N}_{n}^{(n)}\left(J_{n 1}\right)>0\right\}, \quad y \geq \mathrm{l} .
$$

PRomoshrow 3.2. Assume that $\left\{\xi_{n}\right\}$ satisfies $\Delta\left(v_{n}(t)\right)$ for each $\mathrm{t} E T$ and has extremal index $\theta(\mathrm{t})$. Then $\theta(\mathbf{t})=\left(\lim _{i}, \sum_{y=1} y \pi_{n}^{(t)}(y)\right)^{-1}$. 
Proof: Observe that

$$
\begin{aligned}
& \sum_{y \geqslant 1} y \tau_{n}^{(1)}(y)=E\left(\tilde{N}_{n}^{(t)}\left(J_{n 1}\right) \mid \bar{N}_{n}^{(n)}\left(J_{n 1}\right)>0\right)=\frac{r_{n} P\left\{\xi_{1} \pm v_{n}(t)\right\}}{P\left\{\tilde{N}_{n}^{(t)}\left(J_{n 1}\right)>0\right\}}= \\
& \frac{k_{n} r_{n}}{n} \frac{n P\left\{\xi_{1} \neq v_{n}(t)\right\}}{k_{n} P\left\{M_{r_{n}} \equiv v_{n}(t)\right\}} \text {. }
\end{aligned}
$$

Now $\lim _{n \rightarrow \infty} P\left\{\bar{M}_{n} \leftleftarrows v_{n}(\mathbf{t})\right\}=\hat{H}(\mathbf{t})$ and $\quad \lim _{n \rightarrow \infty}$ $P\left\{M_{n} \leq v_{n}(\mathbf{t})\right\}=H(\mathbf{t})$ (by assumption), so that $\lim _{n \rightarrow \mathrm{x}}$ $n P\left\{\xi_{1} \leqslant v_{n}(\mathbf{t})\right\}--\log \hat{H}(\mathbf{t})$ and (by Eq. (13)) $\lim _{n \rightarrow-}$ $k_{n} P\left\{M_{r_{n}} \leqslant v_{n}(\mathbf{t})\right\}=-\log H(\mathbf{t})$. Therefore $\lim _{n \rightarrow \infty} \Sigma_{y i z}$ $y \hat{\pi}_{n}^{(t)}(y)=\log \hat{H}(\mathbf{t}) / \log H(\mathbf{t})-1 / \theta(\mathbf{t})$, as required.

Remark: Proposition 3.2 is simply the multivariate version of Eq. (1) and shows how the extremal index is related to the clustering of "exceedances." Indeed, according to the present viewpoint, an exccedance occurs at timc $i$ if $\xi_{j} v_{n}(t)$, i.c.. if $\xi_{n, j}>v_{n j}\left(t_{j}\right)$ for at least one $j$. Thus Propositions 3.1 and 3.2 show that while the degree of clustering may depend on $t$, it is constant on each $L_{\mathrm{t}}$. Note also the connection to Theorem 2.8 .

The next result gives the relation betwecn the dependence functions of $H$ and $\hat{H}$, which is seen to involve the extremal index in an intricate manner.

Proposition 3.3. If $\left\{\xi_{n}\right\}$ has extremal index $\theta(\mathbf{t}), \mathrm{t} \in T$. then

$$
D_{H}\left(f_{l}^{\theta_{1}}, \ldots, t_{d}^{\theta_{d}}\right)=D_{H}^{(t)}(\mathbf{t}), \quad \mathbf{t} \in T,
$$

where $\theta_{j}$ is the extremal index of $\left\{\xi_{m j}\right\}, j=1, \ldots, d$.

Proof: By definition of the dependence function, $D_{H}(\mathrm{t})-P\left\{H_{1}\left(X_{1}\right) \leq t_{1}, \ldots, H_{d}\left(X_{d}\right) \leq \iota_{d}\right\}$ wherc $\left(X_{1}, \ldots, X_{d}\right)$ is a random vector with distribution $H$. Therefore, since $H_{j}\left(t_{j}\right)=t_{j}^{\theta_{j}}$,

$$
\begin{aligned}
& D_{H}\left(t_{1}^{\theta_{1}}, \ldots, t_{d}^{f_{d}}\right)=P\left\{X_{1} \leq t_{1}, \ldots, X_{d} \leq I_{d}\right\}=H(\mathbf{t})=\hat{H}^{H(t)}(\mathbf{t}) \\
& =D_{H}^{(\mathrm{s})}(\mathrm{t}) \text {, }
\end{aligned}
$$

or required.

\section{REMARKS}

1.) Note that $s=t^{c}$ (for some $>0$ ) if and only if $\log s_{j} / \log$ $s_{j}=\log t_{j} / \log t_{d}-a_{j}$ (say), $j=1, \ldots, d-1$. Therefore we may write $L_{2}-L_{\mathbf{b}}$ where $\mathbf{a}=\left(a_{1, \ldots}, a_{d-1}\right)$, and hence by the remark following Proposition 3.2, $\theta(\mathrm{t})=\theta(\mathrm{a})$, i.e., the extrental index is a function of $d-1$ variables only.

2.) By Proposition 3.3, $D_{H}\left(t_{1}^{\theta_{4}}, \ldots, t_{d}^{\theta_{t}}\right)=D_{H}^{(t) t}(\mathbf{t})=D_{H}\left(\mathbf{t}^{(t)}\right)$. Also, if $\mathbf{t} \in L_{\mathbf{a}}$ then $\left(t_{1}^{\theta_{1}}, \ldots, t_{d}^{\theta_{d}}\right) \in L_{2}$, wherc $\mathbf{a}^{*}=\left(a_{1} \theta_{1} l\right.$ $\left.\theta_{d}, \ldots . . a_{d-1} \theta_{d-1} / \theta_{d}\right)$. Thus $D_{H}$ is obtained by translating the values of $D_{i r}(=\hat{H})$ on $L_{4}$ onto $L_{2}$ *
3.) While the above results illustrate some of the basic properties of the multivariate extremal index, they are far from complete. For instance; it would be useful to identify the set of all "admissible" $\theta(\cdot)$ for a given $\hat{A}$, that is the set of all $\theta(\cdot)$ such that $D_{H}(\cdot)$ defined by Eq. (14) is a probability distribution on $[0,1]^{d}$. It would also be of interest to study the properties of $\theta($.$) when one or both of \hat{H}$ and $H$ are independent. In this context we have the following simple result.

Proposition 3.4. If $\hat{H}$ is independent, then $H$ is independent if and only if

$$
\theta(\mathbf{t})=\sum_{j=1}^{d} \theta_{j} \log t_{j} / \sum_{j=1}^{d} \log t_{j}, \text { for some } \mathbf{t} \in(0,1)^{d} .
$$

In particular, if both $\hat{H}$ and $H$ are independent then $\theta(t)$ is a convex combination of the $\theta_{j}$ 's.

Proor: If $\hat{H}$ is independent, then $H(\mathbf{t})-\hat{H}(\mathbf{t})^{\theta(t)}=$

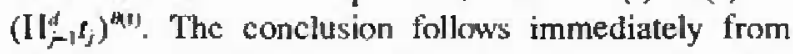
Corollary 2.7 (iii) upon taking logarithms and noting that if $H$ is independent, then $H(t)=\prod_{j-1}^{d} t_{j}^{\phi_{j}}$ -

The extremal index can be given the following more general formulation. Let $\hat{\mu}$ and $\mu$ be the probability measures on $(0,1)^{d}$ corresponding to $\hat{H}$ and $H$, respectively. Thus for instance,

$$
\mu(A)=\lim _{n \rightarrow x} P\left\{M_{n} \in V_{m}(A)\right\}
$$

where $v_{n}(A)-\left\{v_{n}(\mathbf{s}): \mathbf{s} \in A\right\}, A \subset(0,1)^{d}$. We now define $\bar{\theta}(A)$ via the relationship $\mu(A)=\hat{\mu}^{\dot{i}(A)}(A)$, or more directly $\bar{\theta}(A)=\log \mu(A) / \log \hat{\mu}(A)$, for subsets $A \subset(0,1)^{d}$ such that $\hat{\mu}(A)>0$ and $\mu(A)>0$.

Note that $\theta(t)-\bar{\theta}\left(\left(0, t_{1}\right) \times \cdots \times\left(0, t_{d}\right)\right)$ for $t \in T$. Thus if $\{\theta(\mathbf{t}): t \in T\}$ is known along with either of $H$ or $\hat{H}$, then it is possible ut least in theory to obtain $\{\tilde{\theta}(A)$ : $\left.A \subset(0,1)^{d}\right\}$. In practice, however, it may not be possible to obtain $\vec{\theta}(A)$ in a tractable form, but frequently one is only interested in certain special sets, typically rectangles of the form $\mathrm{II}_{j-1}^{d}\left(a_{j}, b_{j}\right)$, and for such sets the computation is easy.

The definition of $M_{n}$ as the vector of componentwise maxima actually corresponds to regarding $\xi_{i}$ as an extreme observation if $\xi_{i}>v_{n j}\left(t_{j}\right)$ for some $j$. More generally, one may define $\xi$ to be an extreme value if $\xi_{i} \in V_{\pi}(A)$ for some $A \subset(0,1)^{d}$, in which case $\bar{\theta}(A)$ has an interpretation as a mcasurc of the clustering of such extremes. Note that the original definition of extremes corresponds to letting $A=\left(\left(0, f_{1}\right) \times \ldots \times\left(0, t_{t}\right)\right)^{c}$. Alternately one may consider taking $A=\left(t_{1}, 1\right) \times \ldots \times\left(t_{d}, 1\right)$ which comesponds to defining $\xi_{l}$ as an cxtreme observa- 
tion if $\xi_{i j}>v_{n j}(t)$ for al] $j$. Yet another choice is $A=$ $\left\{t: \Sigma r_{j}^{2}>c\right\}$.

\section{Examples}

We conclude with two examples, both involving bivariate stationary sequences.

ExAMPLE 4.1 Let $\left\{\eta_{n}\right\}$ be an iid sequence, and put $\xi_{1}=\eta_{n}$, and $\xi_{n 2}=\max \left\{\eta_{n-1}, \eta_{n}\right\}$. Let $F$ denote the distribution of $\xi_{n}-\left(\xi_{n 1}, \xi_{n 2}\right)$ with marginals $F_{1}$ and $F_{2}$. Then $F_{2}(x)=P\left\{\xi_{n 2} \leq x\right\}=P\left\{\eta_{n} \leq x, \eta_{n} \leq x\right\}=F_{\uparrow}^{2}(x)$ and

$$
F\left(x_{1}, x_{2}\right)=P\left\{\xi_{\text {ni }} \leq x_{1}, \xi_{02} \leq x_{2}\right\}= \begin{cases}F_{1}^{2}\left(x_{2}\right), & \text { if } x_{1} \geq x_{2} \\ F_{1}\left(x_{1}\right) F_{1}\left(x_{2}\right), & \text { if } x_{1}<x_{2} .\end{cases}
$$

If $v_{n j}\left(t_{j}\right)$ satisfies $F_{j}^{n}\left(v_{n j}\left(t_{j}\right)\right) \rightarrow t_{j}, j=1,2$, then $\lim _{n \rightarrow \infty}$ $F_{1}^{n}\left(v_{n 2}\left(t_{2}\right)\right)=t_{2}^{1 / 2}$ so that $v_{n 2}\left(t_{2}\right)=v_{n 1}\left(t_{2}^{1 / 2}\right)$. Morcover $v_{n 1}\left(t_{1}\right) \geq v_{n 2}\left(t_{2}\right)$ if and only if $t_{1} \geq t_{2}^{1 / 2}$, and so

$$
H_{n}\left(v_{n}(t)\right)=P\left\{M_{n} \leq v_{n}(t)\right\} \rightarrow-H(t)= \begin{cases}t_{2}^{1 / 2}, & \text { if } t_{1} \geq t_{2}^{1 / 2} \\ t_{1}, & \text { if } t_{1}<t_{2}^{1 / 2}\end{cases}
$$

The marginals of $H$ are thercfore $H_{1}\left(t_{1}\right)=t_{1}$ and $H_{2}\left(t_{2}\right)=$ $t_{2}^{1 / 2}$ so that $\theta_{1}-1$ and $\theta_{2}=1 / 2$, and the depcndence function of $H$ is $D_{H}(t)=H\left(t_{1}, t_{2}^{2}\right)=t_{1} \wedge f_{2}$. For the assuciated itd sequence $\left\{\hat{\xi}_{t}\right\}$ on the other hand, it is easily verified that

$$
\hat{H}_{n}\left(v_{n}(t)\right)=P\left\{\hat{M}_{n} \leq v_{n}(t)\right\} \rightarrow \hat{H}(t)= \begin{cases}t_{2}, & \text { if } t_{1} \geq t_{2}^{1 / 2} \\ t_{1} t_{2}^{1 / 2}, & \text { if } t_{1}<t_{2}^{1 / 2}\end{cases}
$$

from which it follows that

$$
\theta(t)= \begin{cases}\frac{1}{2} t & \text { if } t_{1} \equiv t_{2}^{1 / 2} \\ \frac{\log t_{t}}{\mathrm{~kg} t_{1} t_{2}^{2}=} & \text { if } t_{1}<t_{2}^{1 / 2}\end{cases}
$$

We next consider a moving average sequence studicd in Ref. [16].

EXAMpLE 4.2. Let $\left\{Z_{k}=\left(Z_{k 1}, Z_{k 2}\right)^{\prime}\right\},-x<k<x$, be a scquence of iid random vectors in IR $^{2}$. We assume the existence of a sequence of positive constants $a_{n} \rightarrow x$, and a measure $\nu$ on $1 \mathrm{R}^{2}$ which is finite on sets of the forn] $\{\mathbf{x}:\|\mathbf{x}\|>r\}, r>0$ (where $\|\cdot\|$ denotes the Euclidean norm in $\mathrm{IR}^{2}$ ), such that ${ }^{P} P\left\{a_{n}{ }^{1} Z_{J 0} \in \cdot\right\} \rightarrow{ }^{*} \nu(\cdot)$. (Here ' $\rightarrow$ "' denotes vague convergence of measures on $1 R^{2}$ with respect to the metric $d\left(\mathbf{x}_{3}, \mathbf{x}_{2}\right)=\left|r_{1}^{-1}-r_{2}^{-1}\right| \vee\left|\theta_{1}-\theta_{2}\right|$, where for $i-1,2, r$ and $\theta$, denote the polar coordinates of $\mathbf{x}_{/}$, and $a \vee b=\max \{a, b\}$.) The mcasure $\nu$ is necessirily of the form $\nu(\{\mathbf{x}:\|\mathbf{x}\|>r, \theta(\mathbf{x}) \in A\})=r^{-u} S(A)$ for $r>0$ and $A \subset[0,2 \pi)$, where $S(\cdot)$ is a probability measure on $[0,2 \pi)$ and $\infty>0$. Hence in particular [17],

$$
\nu(c A)=c^{-x} \nu(A)
$$

for all $c>0$ and all sets $A$ with $\nu(A)<\infty$.

Define the bivariale moving average process $\mathbf{X}_{n}=\sum_{-n}^{x} C_{j} \mathbf{Z}_{n j}$, where $\left\{C_{j}-\left|c_{j, j}\right|_{k,-1}^{2}\right\}_{p \geq 0}$ is a sequence of ral $2 \times 2$ matrices satisfying $\Sigma_{j 0}^{x}\left|c_{j k l}\right|^{\delta}<x, k, l=1,2$, for some $\delta \in(0, \alpha)$. $\delta \leq 1$. For $\mathbf{x}=\left(x_{1}, x_{2}\right)^{\prime} \in I^{2}$, writc $A_{y_{y}=}=$ $\left\{z: C_{j} z \in\left(\left(-\infty, x_{1}\right) \times\left(-x_{,}, x_{2}\right)\right)^{c}\right\}$, where $A^{\prime \prime}$ denotes the complement of a set $A \subset \mathrm{IR}^{2}$. Then [16],

$$
\begin{gathered}
\lim _{n \rightarrow \infty} P\left\{a_{n}{ }^{1} \hat{M}_{n} \leq \mathbf{x}\right\}=\exp \{-\dot{\gamma}(\mathbf{x})\}, \text { and } \\
\lim _{n \rightarrow \infty} P\left\{a_{n}^{-1} M_{n} \leq \mathbf{x}\right\}-\exp \{-\gamma(\mathbf{x})\}, \mathbf{x} \in \mathbb{I R}^{2},
\end{gathered}
$$

where $\hat{\gamma}(x)-\Sigma_{j 0}^{x} \nu\left(A_{y_{j}}\right)$ and $\gamma(x)=\nu\left(U_{j, j}^{x} A_{, j}\right)$. The extremal inctex is therefore $\theta(\mathbf{x})=\gamma(\mathbf{x}) / \hat{\gamma}(\mathbf{x}), \mathbf{x} \in \mathbb{R}^{2}$. It follows from the definition of $A_{x, j}$ and Eq. (15) that this is in fact a function of $x_{1} / x_{2}$. Note that the extremal index defined above differs from that in Sec. 3 in that it is defined on $\mathbb{I R}^{2}$ rather than $[0.1]^{2}$. However the two defimitions arc equivalent as may be seen by means of a suitabic transformation from $\left[R^{2}\right.$ to $[0,1]^{2}$.

The actual calculation of $\theta(\mathbf{x})$ may be quite difficult in general, but possible to carry out under appropriate simplifying assumptions.

Case (i). If $C_{j}-c_{j} C$ where $C=\left[c_{u}\right]_{k j-1}^{2}$ and the $c_{j}{ }^{\prime} \mathrm{s}$ are non-negative constants, then $A_{x_{j}}=c_{j}^{-1} B(x)$ and

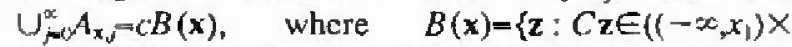
$\left.\left.\left(-x_{,} x_{2}\right)\right)^{\prime}\right\}$ and $c=\max \left\{c_{j}: j \geq 0\right\}$. Therefore by Eq. (15), $\nu\left(A_{\mathrm{x}, j}\right)=c_{j}^{u} \nu(B(\mathrm{x}))$ and $\nu\left(\bigcup_{j=0}^{\pi} A_{\mathbf{x}, j}\right)=c^{u} \nu(B(\mathrm{x}))$ so that $\theta(\mathbf{x})=c^{\sigma} / \mathbf{I}_{j-1}^{\infty} c_{j}^{\alpha}$.

Case (ii). If the $C_{j}$ s are diagonal, i.e.. $C_{j}=\operatorname{diag}\left[c_{j 1}, c_{j 2}\right]$ with $c_{j} \geq 0, i=1,2$, then $A_{x, j} \sim\left\{\mathrm{z}: c_{j 1} z_{1}>x_{1}\right.$ or $\left.c_{j 2} \bar{z}_{2}>x_{2}\right\}$ and $\bigcup_{-1, A_{x, j}}^{\infty}=\left(\left(-\infty, x_{1} / c_{1}\right) \times\left(-\infty, x_{2} / c_{2}\right)\right)^{c}$ where $c_{1}=$ $\max \left\{c_{j *} ; j \geq 0\right\}, i=1,2$, In particular, taking $x_{2}=x$ and using Eq. (15) as in Case (i), we have $\nu\left(A_{\pi_{j}}\right)=$

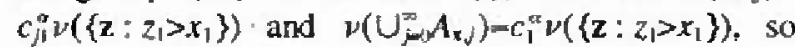
that the extremal index of $\left\{X_{n 1}\right\}$ is $\theta_{1}=c_{1}^{\alpha} / \Sigma_{j=0}^{\infty} c_{j=1}^{\mu}$.

Case (iii). Let $D$ denotc the support of $\nu$. If $D C$ $\left\{z: z_{1}=0\right.$ or $\left.z_{2}=0\right\}$ (which is the case if the coordinates of $\mathbf{Z}_{0}$ are indcpendent), then we may write

$$
\nu\left(\left(-x_{,}, x_{1}\right) \times\left(-x_{1}, x_{2}\right)\right)^{c}=a_{1} x_{1}^{-\alpha}+a_{2} x_{2}{ }^{\alpha}, \quad x_{1}, x_{2} \geq 0 .
$$

for suitablc constants $a_{1} \equiv 0$ and $a_{2} \equiv 0$. Oncc again, assuming the $c_{j, k i}$ 's to be non-regative and writing $c_{k i}=\max \left\{c_{j k i}: j \geq 0\right\}$ for $k . l=1,2$, we have (writing $a \wedge b=\min \{a, b\})$

$$
A_{2, j} \cap D=\left(\left(-x_{-} x_{1} / c_{j .11} \wedge x_{2} / c_{j, 21}\right) \times\left(-x_{-x_{1}} / c_{j .12} \wedge x_{2} / c_{j, 22}\right)\right) \cap D
$$


and

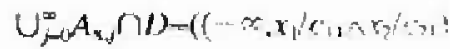

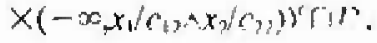

so that using Eq. (16)

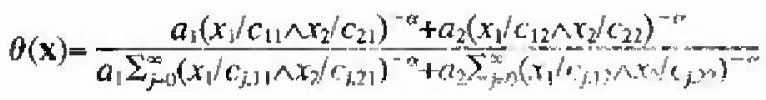

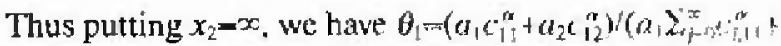

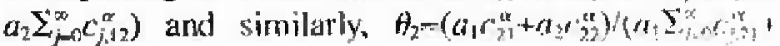
$\left.a_{2} \Sigma_{j=1}^{\infty} c_{j, 22}^{a}\right)$.

If also $c_{j, 2}-c_{j \geq 1}=0$ for cach $j$, (that is if the $r j$, ant? diagonal), then

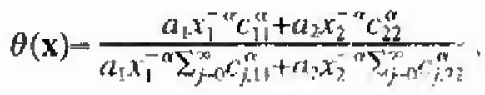

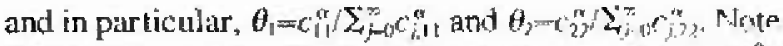
that in this case the limiting distributions of $M_{n}$ arm $\hat{M}_{n}$ are both independent, and hence (in arcordance with Proposition 3.4) $\theta(\mathbf{x})$ is a onvex combination of $y_{1}$ an! $\theta_{2 *}$

The non-negativencss of the $C$, $s$ assumed alym is not crucial and may be relaxed, although ot the remer at more involved calculations.

\section{Acknowledgments}

It is a pleasure to thank Frofessor M. R. Learbetter for his encouragement and guidance during the course. of this research. Part of this work was carried out ruring a brief visit to the University of Bern (supported tyy the Swiss National Science Foundation), ard I am very grateful to Professor J. Hüsler for several aseful discus sions. Research supported by the Air Force Office af Scientific: Research Contract No. F49620 850 (01/1.

\section{References}

I1] M. R. Leadhetter, Extremes and local drpendence in a station? y sequence, Z. Wabr-sch. verw. Gebietc. 65, 291-30) (1083)

[2] G. F. Newell. Asymptolic extrethes for m-depentern tondom variablcs. Ann. Math. Statist. 35, 1327-3 325 (1964)

[3] R. M. Loynes, Extreme values in unifornly rixing "ruplbitif: processes. Ann. Math. Statist. 36, 993 -999 (1965).

[4] G. L. O'Brien. The naximum term of Iniforraly mit wing stathy? ary processes. Z. Watr-sch. verw. Gebiete, 30. 57.69 (1971)

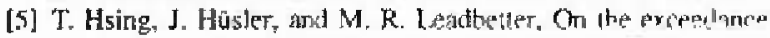

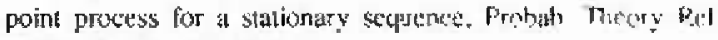
Fields. 78, 97-1/2 (1988)
[6] E. de Hitart and S. Resnick. Limit theory for multivariate sample "xt rembs. Z. Wahtsch. verw. Gebicte. 40, 317-337 (1977).

(7) A. W Marshall and 1. Olkin, Domains of attraction of multivariate eximus valus distributions, Ann. PTobab. 11, 168-177 (198.3).

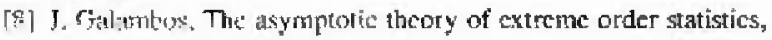
Wildy. New York (1987).

[9] T. Hsing, Extreme value theory for multivariate slationary sequences. 1. Multivariale Ánal. 29, 274-29) (1989).

[It!] I. Hüs|nr. Mulliwariate cxireme values in stationary mandon sequences. Siochastic Proc. Appl. 35, 99-108 (1990).

|' ' l fi: Leheuvels, Caractèrisation complete Jes lois extrêmes mulitvatiécs et de la crnnutgenue des types exlrêmes. Publ, Instit. Sirtis. Univ. Paris. 23, 1-36 (1978).

[1?.] S. Ningtagopilan. M. R. Leadbetter, and J. Hussler. Limit theorems fer rulíf-dimensional randnm measures. 92-14, Departmoll "Il' Stutistics. Colorado State University, November 1992.

(I 11 M. R. Leadbetter. (1974). On extreme values in stationary sequnces, $Z$. Wahrsch. verw. Gebiete. 28. 298-303 (1974).

IIA.I R. Takahashi, Sone properties of multivariate exlreme value dietrihuting an! multivariate tail equivalence, Ann. Inst. Statist. Mith. 39. 537-647 (1987).

115] N Catkan. University of Bern, Privale communication, (1993),

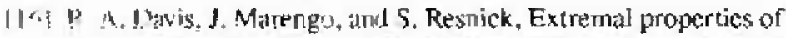
it L a diss of multitutiale mowizg averages, L1-4, Procedings of the 45th Sersion of the 1.S.I.. Amsterdam, (19R.5) pp. 1--14.

11:। S. kasniuk, Extreme values, regular variation, and poin procerses, Springer-Verlag. New York (1987).

Ahowt the outhor: \& Nandagopalan is an Assistant

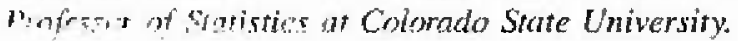

